\title{
One- and two-center physical space partitioning of the energy in the density functional theory
}

\author{
P. Salvador ${ }^{\text {a) }}$ \\ Department of Chemistry, University of Girona, 17071 Girona, Spain and Institute of Computational \\ Chemistry, University of Girona, 17071 Girona, Spain \\ I. Mayer \\ Chemical Research Center, Hungarian Academy of Sciences, P.O. Box 17, H-1525 Budapest, Hungary
}

(Received 8 March 2007; accepted 24 April 2007; published online 19 June 2007)

\begin{abstract}
A conceptually new approach is introduced for the decomposition of the molecular energy calculated at the density functional theory level of theory into sum of one- and two-atomic energy components, and is realized in the "fuzzy atoms" framework. (Fuzzy atoms mean that the three-dimensional physical space is divided into atomic regions having no sharp boundaries but exhibiting a continuous transition from one to another.) The new scheme uses the new concept of "bond order density" to calculate the diatomic exchange energy components and gives them unexpectedly close to the values calculated by the exact (Hartree-Fock) exchange for the same Kohn-Sham orbitals. @ 2007 American Institute of Physics. [DOI: 10.1063/1.2741258]
\end{abstract}

\section{INTRODUCTION}

In the past years, there has been a growing interest in the so-called molecular energy decomposition schemes. With such a posteriori analyses of the results obtained in the quantum chemical calculations, the total energy of a system is expressed (exactly or approximately) as a sum of atomic and diatomic terms. The atomic energy terms, as compared with the energies of the free atom, account for the gain or loss of intra-atomic energy upon bond formation, whereas the diatomic terms naturally reflect the energetic aspects of the intramolecular interactions present in the molecule. As the individual atoms within a molecule are not true quantum mechanical observables, there are different methods to define them approximately, leading to different schemes of analysis: it can be accomplished either in the Hilbert space of atomcentered basis orbitals or directly in the thee-dimensional (3D) physical space. ${ }^{1}$ For the Hilbert-space analysis, energy decomposition schemes of the Hartree-Fock (HF) and density functional theory (DFT) energy have already been devised and successfully applied. ${ }^{4-6}$ These schemes are obviously restricted to basis sets having true atomic character and become ill defined in the complete basis set limit. In order to carry out a decomposition in the physical space, one must assign in some sense a part of the 3D space to each atom. The analysis of different quantities in the 3D space is most often accomplished by applying the atoms in molecules (AIM) theory, ${ }^{7}$ defining the so-called atomic basins. In our study, ${ }^{8}$ the decomposition of the Hartree-Fock energy in the AIM framework led to results which are in good qualitative agreement with those of the respective Hilbert-space decomposition. ${ }^{4}$ However, the cumbersome numerical twoelectron (i.e., formally six dimensional) integrations are extremely CPU demanding, basically due to the complex shape

\footnotetext{
${ }^{a)}$ Electronic mail: pedro.salvador@udg.es
}

of the atomic basins. For each atom or atomic pair, there is one such integration for calculating the Coulomb energy contribution and one for each pair of occupied molecular orbitals for the respective exchange energy component. Blanco et al. ${ }^{9}$ have extended the Hartree-Fock energy decomposition scheme of Ref. 10 to the post-Hartree-Fock case by partitioning the second order density matrix and introducing an efficient numerical quadrature algorithm for the two-center integrations. Despite of the effectiveness of their integration algorithm, it is still not sufficient for routine calculations of larger systems, while population analysis and bond order calculations are feasible.

Most recently, we have proposed, ${ }^{11,12}$ as an alternative to AIM, the use of "fuzzy atoms" for the 3D analysis. Fuzzy atoms mean that the 3D physical space is divided into atomic regions having no sharp boundaries but exhibiting a continuous transition from one to another. Such "fuzzy" atoms were first considered by Hirshfeld ${ }^{13}$ and recently explored by Clark and Davidson ${ }^{14,15}$ for calculating effective atomic charges in molecules by using the so-called stockholders scheme; our definition is different only by use of another weight function (that of Becke ${ }^{16}$ ) for calculating the distribution of the electron density between the different atoms at different points. The advantages of using fuzzy atoms are basically twofold. First, the numerical integrations are much cheaper computationally, which is especially important for the two-electron ones. Second, since they have no sharp boundaries, the atoms share the interatomic part of the physical space to some extent. This allowed us ${ }^{11,12}$ to introduce the concept of overlap population in the 3D framework, as well as to modify our previous formalism-actually to regroup some kinetic energy components-and devise an energy decomposition scheme of the Hartree-Fock energy as to get diatomic energy components which are "on the chemical scale." 17 (In this respect, the fuzzy atoms 3D decomposition scheme is much similar to the Hilbert-space analysis where 
one obtains diatomic kinetic energy terms quite naturally.) Energy components are static parameters computed from the actual molecular wave function, they are not directly related to the dissociation energies, and, therefore, there is no requirement that they must be on the "chemical scale." Nevertheless, having such components which compare well with the dissociation energies seems to be desirable from a chemist's point of view. (For a summary of our approaches to the problem, we refer to Ref. 18; in Ref. 19, a dilemma connected with the use of diatomic kinetic energy component is discussed in detail.)

It is worth mentioning that it is relatively easy to introduce a general scheme for the partitioning of functions such as the electron density, obtained at any level of theory. However, when dealing with the decomposition of the molecular energy, one faces the difficulty that the energy formulas of different methods contain quite different types of quantities, and hence, it is not easy to devise a general strategy to treat all levels of theory on equal footing. Since the basic idea behind the method is to decompose each term that contributes to the total energy, we are forced to apply somewhat different strategies for different quantum chemical methodologies.

In a recent paper, ${ }^{6}$ it has been shown that the results of the Hilbert-space Hartree-Fock energy component analysis ${ }^{5}$ can also be formulated by introducing effective atomic first order density matrices $\rho^{A}\left(r, r^{\prime}\right)$ and effective atomic potentials $\hat{V}^{A}$ (having a nonlocal component accounting for exchange); then the electron-nuclear and electron-electron interaction energies can be presented as a sum of integrals representing effective atomic potentials acting on the different effective atomic density matrices. Along that line, a Hilbert-space decomposition of the DFT energy has also been proposed ${ }^{6}$ based on the approximate expansion of the exchange-correlation energy density per electron in terms of the basis orbitals. Reasonable energy components have been obtained for different DFT functionals, although sensitive to the variations of the method and, in particular, to the geometry variations taking place when one turns from HF to different DFT variants. That may indicate that we have not yet reached the ultimate solution even for that Hilbert-space DFT energy decomposition problem. Our attempts to get a straightforward generalization of the methods used in the Hartree-Fock case to the fuzzy atom DFT energy decomposition did not give satisfactory results at all. In fact, one could anticipate that if one uses the same simple direct approaches in DFT which were successful in HF, then one may get difficulties: there is no obvious way to decompose the exchange energy with a density-based local exchange functional in the same straightforward manner as it is done in the Hartree-Fock case. The reason is that one cannot exactly determine how much exchange energy is gained by atom $A$ due to the density coming from atom $B$, because the exchange-correlation functionals are nonlinear.

Despite of all these problems, we felt rather desirable to extend the 3D energy decomposition method and devise a general decomposition scheme for the DFT energy in terms of atomic and diatomic contributions. In fact, the bottleneck of the 3D-space energy decompositions for wave-function- based methods such as HF is the computation of the exchange energy contributions, since they involve extremely time consuming numerical integrations for each pair of atoms and each pair of occupied orbitals. Pure generalized gradient approximation methods (without any admixture of the "exact" HF exchange) are of practical interest since they require only a single two-electron integration per pair of atoms (for the Coulomb energy component), thus offering us the possibility of energy decompositions which are affordable in routine calculations. Hence we focus on this particular family of DFT functionals and do not consider those DFT variants (B3LYP, OEP, etc.) in which exchange is computed (fully or partly) by using the exact Hartree-Fock exchange energy formula.

In what follows, we will show that one can get an appropriate DFT energy decomposition scheme on the basis of recalling the role of exchange in the chemical bond formation.

\section{PHYSICAL-SPACE DECOMPOSITION OF THE DFT ENERGY}

\section{A. Atomic decomposition of identity and the energy components}

Recently, a scheme of "atomic decomposition of identity" has been introduced ${ }^{2}$ in which the identity operator is written as a sum of terms ${ }^{20}$ which are-in that or another manner-assigned to the individual atoms $A$ :

$$
\hat{I}=\sum_{A} \hat{\rho}_{A} .
$$

This permits us to treat the most different schemes of population analysis and energy decomposition in a framework of a common formalism. In the case of fuzzy atoms, the decomposition is based on assigning a non-negative weight function $w_{A}(\mathbf{r})$ to each atom in every point $\mathbf{r}$ of the $3 \mathrm{D}$ space, satisfying the requirement

$$
\sum_{A} w_{A}(\mathbf{r})=1
$$

everywhere. It is assumed that $w_{A}(\mathbf{r})$ is large "inside" atom $A$ and quickly becomes negligible outside.

The decompositions pertinent to the fuzzy atoms can be obtained by defining the operators $\hat{\rho}_{A}$ as

$$
\hat{\rho}_{A}=\left.w_{A}\left(\mathbf{r}^{\prime}\right)\right|_{\mathbf{r}^{\prime}=\mathbf{r}} .
$$

Here the notation $\mathbf{r}^{\prime}=\mathbf{r}$ indicates that one should replace $\mathbf{r}^{\prime}$ by $\mathbf{r}$ after the action of all the operators on the wave functions depending on $\mathbf{r}$ is evaluated, but before the integration over $\mathbf{r}$ is carried out. Thus quantum mechanical operators act only on the electronic wave functions but not on the weight functions $w_{A}(\mathbf{r})$. In the particular case of the AIM framework, the atomic weight functions take the value of 1 if the point belongs to the basin of the atom and 0 otherwise.

By using this formalism of the "atomic resolution of identity" and the definition [Eq. (3)] of the operators $\hat{\rho}_{A}$, one can easily recover the formulas used in Refs. 8 and 11 for the decomposition of the HF energy within AIM and fuzzy atom frameworks, respectively. The two schemes of calculations 
discussed in the latter paper differ by introducing the decomposition [Eq. (2)] once or twice in the expectation value of the kinetic energy operator $\hat{T}$. In the first case, the kinetic energy is decomposed into atomic contributions only; in the second one, it is presented as a sum of mono- and diatomic contributions.

The expressions of all the energy components are the same in the HF and DFT cases, except the exchange (exchange-correlation) energy. Thus, assuming a closed-shell case with doubly filled orbitals, we have for the kinetic energy $E_{\text {kin }}$ either the expansion

$$
E_{\mathrm{kin}}=-\sum_{A} \sum_{i}^{\mathrm{occ}} \int w_{A}(\mathbf{r}) \varphi_{i}^{*}(\mathbf{r}) \Delta \varphi_{i}(\mathbf{r}) d v,
$$

representing a sum of terms of atomic type, or the expression

$$
E_{\mathrm{kin}}=-\sum_{A, B} \sum_{i}^{\mathrm{occ}} \int w_{A}(\mathbf{r}) w_{B}(\mathbf{r}) \varphi_{i}^{*}(\mathbf{r}) \Delta \varphi_{i}(\mathbf{r}) d v
$$

which is a sum of monoatomic $(A=B)$ and diatomic $(A$ $\neq B)$ terms.

Similarly, the electron-nuclear attraction energy

$$
E_{\mathrm{el}-\mathrm{nuc}}=-2 \sum_{A, B} \sum_{i}^{\mathrm{occ}} \int w_{A}(\mathbf{r}) \frac{Z_{B}}{r_{B}}\left|\varphi_{i}(\mathbf{r})\right|^{2} d v
$$

and the Coulomb energy

$$
\begin{aligned}
E_{\text {Coul }}= & 2 \sum_{A, B} \sum_{i, j}^{\text {occ }} \iint w_{A}\left(\mathbf{r}_{1}\right) w_{B}\left(\mathbf{r}_{2}\right) \\
& \times\left|\varphi_{i}\left(\mathbf{r}_{1}\right)\right|^{2} \frac{1}{r_{12}}\left|\varphi_{j}\left(\mathbf{r}_{2}\right)\right|^{2} d v_{1} d v_{2}
\end{aligned}
$$

both contain monoatomic and diatomic components depending on $A=B$ or $A \neq B$. (The nuclear repulsion energy is obviously a sum of diatomic terms only.)

The exchange-correlation energy (functional) is an integral over the exchange-correlation energy density $\varepsilon_{x c}[\rho(\mathbf{r})]$; thus, formally, it is a one-electron quantity, for which both decompositions such as Eqs. (4) and (5) would be possible, giving

$$
E_{\mathrm{xc}}=\sum_{A} \int w_{A}(\mathbf{r}) \varepsilon_{\mathrm{xc}}[\rho(\mathbf{r})] d v
$$

and

$$
E_{x c}=\sum_{A, B} \int w_{A}(\mathbf{r}) w_{B}(\mathbf{r}) \varepsilon_{x c}[\rho(\mathbf{r})] d v,
$$

respectively. However, as noted in the Introduction, the nonlinear character of the exchange functional indicates that this avenue is questionable from a physical point of view. Also, our numerical studies showed that these formulas do not give reasonable results from a chemical point of view either, because the exchange appears basically attributed to the atoms and not to the bonds. Furthermore, no diatomic exchange energy components would be obtained for disjoint atomic domains such as AIM theory, which can hardly be put in correspondence with bonding interactions. Therefore, the de- composition of the exchange energy requires another approach,

\section{B. Bond order density and exchange energy components}

The pair density (diagonal part of the spinless second order density matrix) for a single determinant wave function is $^{21}$

$$
\varrho_{2}\left(\mathbf{r}, \mathbf{r}^{\prime}\right)=\varrho(\mathbf{r}) \varrho\left(\mathbf{r}^{\prime}\right)-\varrho^{x}\left(\mathbf{r}, \mathbf{r}^{\prime}\right),
$$

where the first ("direct" or "Coulomb type") term is the product of the electron densities in points $\mathbf{r}$ and $\mathbf{r}^{\prime}$, whereas the second is the two-electron "exchange density." The exchange density originates from the antisymmetry of the wave function; it also contains the compensation for the "selfinteraction" of the electrons. ${ }^{22}$ If one uses doubly occupied orthonormalized orbitals (restricted Hartree-Fock or closedshell Kohn-Sham determinants), then the exchange density can be expressed via the molecular orbitals $\varphi_{i}$ as $^{21}$

$$
\varrho^{x}\left(\mathbf{r}, \mathbf{r}^{\prime}\right)=2 \sum_{i, j}^{\mathrm{occ}} \varphi_{i}^{*}(\mathbf{r}) \varphi_{j}^{*}\left(\mathbf{r}^{\prime}\right) \varphi_{j}(\mathbf{r}) \varphi_{i}\left(\mathbf{r}^{\prime}\right) .
$$

Integrating over both coordinates and taking into account the orthonormalization of the orbitals, one gets the normalization of the exchange density as $^{21}$

$$
\iint \varrho^{x}\left(\mathbf{r}, \mathbf{r}^{\prime}\right) d v d v^{\prime}=N,
$$

where $N$ is the number of electrons.

Now, introducing in the integrand of Eq. (12) two atomic resolutions of identity as $\Sigma_{A} \Sigma_{B} w_{A}(\mathbf{r}) w_{B}\left(\mathbf{r}^{\prime}\right)=1$, we get the decomposition of this normalization of the exchange density into atomic $(A=B)$ and diatomic $(A \neq B)$ contributions:

$$
\sum_{A, B} \iint w_{A}(\mathbf{r}) w_{B}\left(\mathbf{r}^{\prime}\right) \varrho^{x}\left(\mathbf{r}, \mathbf{r}^{\prime}\right) d v d v^{\prime}=N .
$$

The two terms of this sum, corresponding to a given pair of atoms $A$ and $B$, equal the bond order index $B_{A B}$ between them, defined in the fuzzy atom framework:

$$
\begin{aligned}
B_{A B}= & \iint w_{A}(\mathbf{r}) w_{B}\left(\mathbf{r}^{\prime}\right) \varrho^{x}\left(\mathbf{r}, \mathbf{r}^{\prime}\right) d v d v^{\prime} \\
& +\iint w_{B}(\mathbf{r}) w_{A}\left(\mathbf{r}^{\prime}\right) \varrho^{x}\left(\mathbf{r}, \mathbf{r}^{\prime}\right) d v d v^{\prime} .
\end{aligned}
$$

(It is easy to check that this definition is equivalent to the explicit one given in Ref. 12.) Thus the bond order can be considered as the integral over the diatomic part of the exchange density matrix,

$$
\varrho_{A B}^{x}\left(\mathbf{r}, \mathbf{r}^{\prime}\right)=w_{A}(\mathbf{r}) w_{B}\left(\mathbf{r}^{\prime}\right) \varrho^{x}\left(\mathbf{r}, \mathbf{r}^{\prime}\right)+w_{B}(\mathbf{r}) w_{A}\left(\mathbf{r}^{\prime}\right) \varrho^{x}\left(\mathbf{r}, \mathbf{r}^{\prime}\right),
$$

which, at the Hartree-Fock level of theory, gives rise to the Hartree-Fock exchange energy component 


$$
E_{x, A B}^{\mathrm{HF}}=-\frac{1}{2} \iint \frac{\varrho_{A B}^{x}\left(\mathbf{r}, \mathbf{r}^{\prime}\right)}{\left|\mathbf{r}-\mathbf{r}^{\prime}\right|} d v d v^{\prime}
$$

The diatomic exchange energy components represent large negative numbers for chemically bonded atoms, usually correlating well with the respective bond order indices. ${ }^{23}$

It was shown by Borisova and Semenov ${ }^{25}$ for the Wiberg index $^{26}$ of the complete neglect of differential overlap (CNDO) theory and by one of us for the $a b$ initio $^{27}$ that the bond order index calculated in the Hilbert-space framework may be considered as the effective number of electron pairs participating in the bonding between the atoms considered. ${ }^{28}$ This should be applicable mutatis mutandis in the fuzzy atoms frame as well.

It is important to realize that neither the bond order index nor the Hartree-Fock exchange energy components are directly related to the interatomic overlap populations, i.e., to the electronic charge accumulated in the bonding regions. They are connected with the existence of bonding molecular orbitals spreading over the atoms in question, which leads to a correlation between the fluctuations of the atomic populations: ${ }^{18,29}$ if there is a positive charge fluctuation on one atom, then on the bonded atom, a negative one may be expected-and this correlation leads to a negative energy contribution, similar to that as the correlation of fluctuating dipoles causes attractive dispersion interaction. This negative energy component appears even if the charge is completely concentrated near the nuclei: it is present ${ }^{18,30,31}$ in the CNDO-type semiempirical theories in which the overlap populations integrate to zero (the orbitals are assumed orthogonal) and the zero differential overlap approximation is used for the integrals, and even in the case of a point-charge approximation of the two-electron integrals. ${ }^{31,32}$

This connection between the diatomic exchange, bond orders, and negative exchange energy contributions motivated us to introduce a new quantity called "bond order density" - it will be discussed in more detail elsewhere. The bond order density $\beta_{A B}(\mathbf{r})$ for the pair of atoms $A$ and $B$ is obtained by integrating the diatomic exchange density $\varrho_{A B}^{x}\left(\mathbf{r}, \mathbf{r}^{\prime}\right)$ over the coordinate $\mathbf{r}^{\prime}$; for the closed-shell case considered here, one gets

$$
\beta_{A B}(\mathbf{r})=2 \sum_{i, j}^{\text {occ }}\left(w_{A}(\mathbf{r}) S_{i j}^{B}+w_{B}(\mathbf{r}) S_{i j}^{A}\right) \varphi_{i}^{*}(\mathbf{r}) \varphi_{j}(\mathbf{r}),
$$

where the elements of the "atomic overlap matrix" $\mathbf{S}^{A}$ of the molecular orbitals are defined as

$$
S_{i j}^{A}=\int w_{A}(\mathbf{r}) \varphi_{i}^{*}(\mathbf{r}) \varphi_{j}(\mathbf{r}) d v .
$$

The name bond order density has been proposed because this function integrates to the bond order index between the pairs of atoms $A$ and $B$. Actually, it represents the part of the total electron density that is used by the bonding interaction between atoms $A$ and $B$ through the exchange interaction. For $A=B$, the intra-atomic bond order density $\beta_{A A}$ can also be defined; it stands for the part of the electron density of atom $A$ that is not involved in the bonds: ${ }^{33}$

$$
\beta_{A A}(\mathbf{r})=2 \sum_{i, j}^{\text {occ }} w_{A}(\mathbf{r}) S_{i j}^{A} \varphi_{i}^{*}(\mathbf{r}) \varphi_{j}(\mathbf{r})
$$

Equation (17) is the definition equation of the bond order density for the single determinant wave functions used in the Hartree-Fock or Kohn-Sham frameworks. Obviously, in the DFT case, the exchange density equation [Eq. (11)] and thus the bond order density equation [Eq. (17)] reflect the pair density properties of the Kohn-Sham single determinant wave function and should be considered as being only some approximations from the point of view of an exact (orbitalfree) DFT theory.

One of the important features that concerns the present case is that the sum of all atomic and diatomic bond order densities exactly reproduces the molecular electronic density, so that

$$
\varrho(\mathbf{r})=\sum_{A \leqslant B} \beta_{A B}(\mathbf{r})=\sum_{A} \beta_{A A}(\mathbf{r})+\frac{1}{2} \sum_{\substack{A, B \\ A \neq B}} \beta_{A B}(\mathbf{r}) .
$$

The above considerations mean that the introduction of the concept of bond order density permits us to decompose the electronic density in a manner reflecting closely the bonding interactions between the atoms. This decomposition differs from all the standard ones. Figure 1 depicts the diatomic bond order density (henceforth BOD) along the bond axis of the $\mathrm{CO}$ molecule calculated at the BLYP/6-31G ${ }^{* *}$ level. It has two peaks at the atomic positions but also extends into the interatomic region resembling a bonding orbital. In particular, it is important to stress that the electron density actually involved in the bond is not solely localized in the interatomic region but is partially concentrated also in the regions close to the nuclei. This observation is in full agreement with the discussion above about the character of the exchange interaction and stresses again the differences between bond orders and overlap populations.

Another important point is that (contrary the overlap populations) the BOD does not vanish when nonoverlapping atomic domains are used. Thus the present scheme is applicable to any method of decomposing the 3D space, including AIM or the use of Voronoi cells. In Fig. 2, we show the BODs for the $\mathrm{CO}$ bond calculated from three different types of atomic partitionings, namely, Becke, AIM, and Becke-rho. The latter ${ }^{12,37,38}$ stands for Becke's fuzzy atom weights calculated using the minimum of the electron density along the interatomic distance to define the ratio between the atomic radii of the two atoms, which is to be used in the Becke formula ${ }^{16}$ for calculating the weight functions $w_{A}(\mathbf{r})$. It has been shown recently ${ }^{37}$ that the AIM and Becke-rho schemes yield very similar populations and bond orders. In all cases, the BODs exhibit the two characteristic peaks at the atomic positions and extends over the interatomic region. By comparing the Becke and Becke-rho functions, one can see that the difference in atomic sizes affect the height of the atomic peaks and the polarization of the BOD in the interatomic region. The main feature of the AIM BOD is that it is not continuous (except for the special case of symmetric homonuclear bonds), which complicates its topological analysis. Apart from that, the shape of the BOD obtained for different 


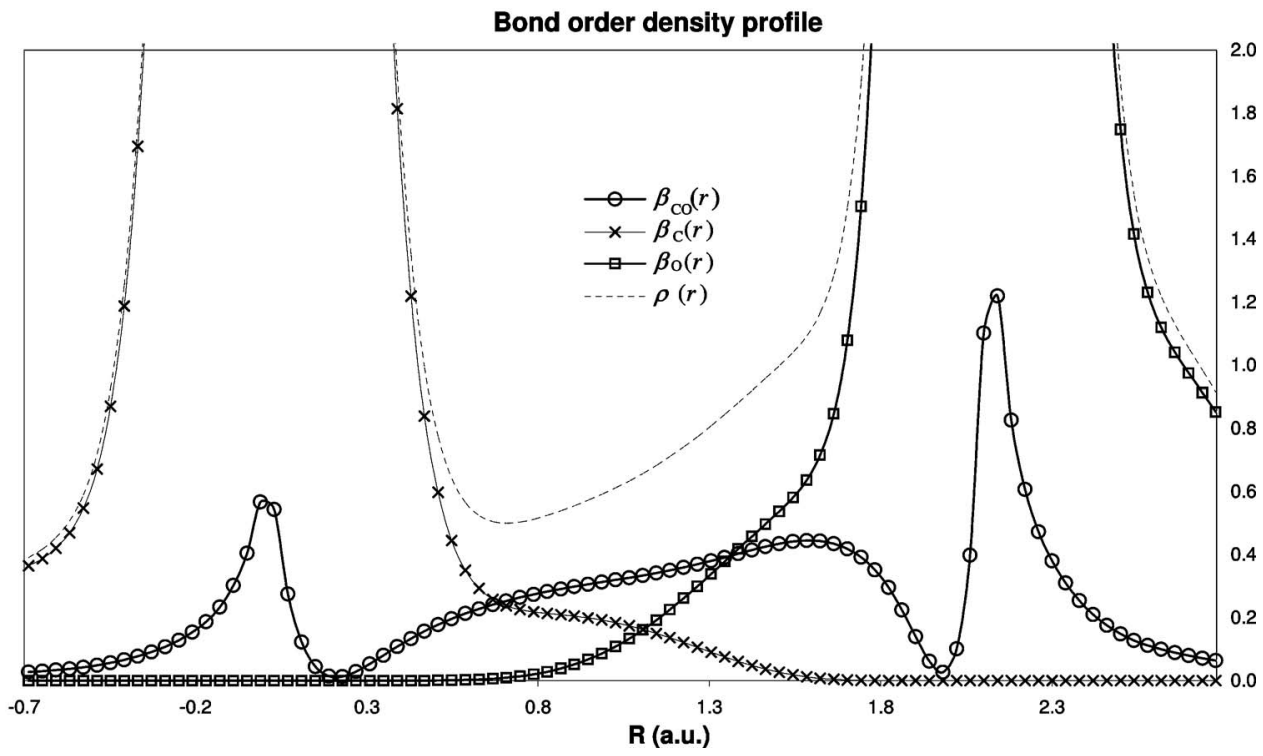

FIG. 1. Bond order density, intraatomic bond order densities, and total electron density profiles (in a.u.) for the $\mathrm{CO}$ molecule along the interatomic axis from BLYP/6-31G ${ }^{* *}$ Kohn-Sham orbitals. physical-space partitionings are quite similar, which is to be translated in the corresponding values of the diatomic exchange energy contributions.

If in Eq. (16) one performs the integration only over $\mathbf{r}^{\prime}$, then one gets an expression for the diatomic Hartree-Fock exchange energy density as follows:

$$
\varepsilon_{x, A B}^{\mathrm{HF}}(\mathbf{r})=-\frac{1}{2} \int \frac{\varrho_{A B}^{x}\left(\mathbf{r}, \mathbf{r}^{\prime}\right)}{\left|\mathbf{r}-\mathbf{r}^{\prime}\right|} d v^{\prime}
$$

It is not surprising (see Fig. 3) that its topology-after changing the sign - is very similar ${ }^{39}$ to that of the bond order density $\beta_{A B}(\mathbf{r})$. This motivated us to suppose that the energetic contribution of the BOD could be determined by using the local exchange energy density functionals of the DFT. That is, we introduce the bond order density given by Eq. (17) into the DFT energy expression $E_{x}=\int \varepsilon_{x}[\varrho(\mathbf{r})] d v$ and get the definition for the diatomic exchange energy component as

$$
E_{x, A B}=\int \varepsilon_{x}\left[\beta_{A B}(\mathbf{r})\right] d v
$$

We have calculated these diatomic exchange energy components within the fuzzy atom framework at the BLYP/6-31G ${ }^{* *}$ level of theory ${ }^{40}$ and used the same numerical integration scheme ${ }^{41}$ as that in Ref. 11. The results obtained for the relevant bonding interactions in a selected set of 12 molecules are listed in Table I. (As known, the BLYP scheme uses the Becke88 functional for the exchange.) The results obtained are in surprisingly good agreement with the Hartree-Fock ones - obtained with the same Kohn-Sham orbitals at the same DFT geometry. The correlation between the two sets of values is almost perfect, with a slope very close to unity (see Fig. 4). This is especially the case if one does not include the multiply bonded molecules $\mathrm{CO}$ and $\mathrm{N}_{2}$ having very large exchange contributions and distorting somewhat the regression (see Fig. 4). The correlation equa-

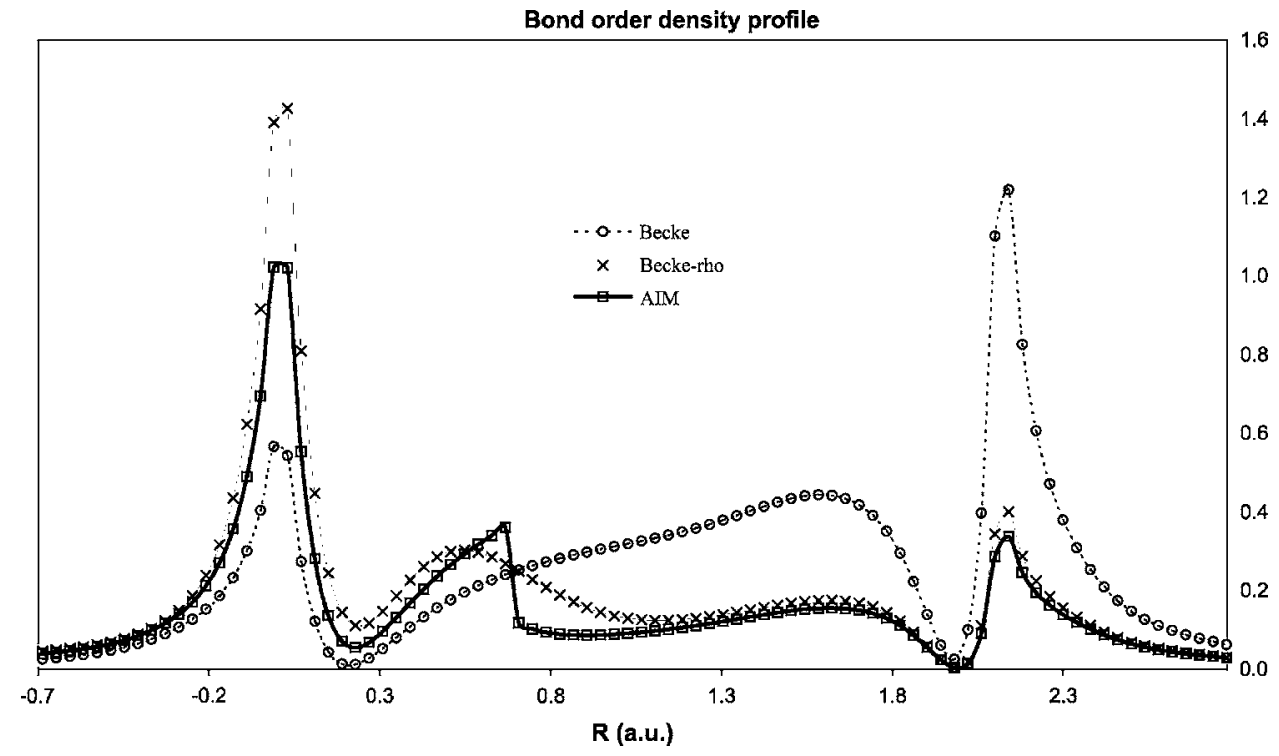

FIG. 2. Bond order density profiles for several atomic definitions (see text) along the intermolecular axis of the CO molecule from BLYP/6-31G** Kohn-Sham orbitals. 


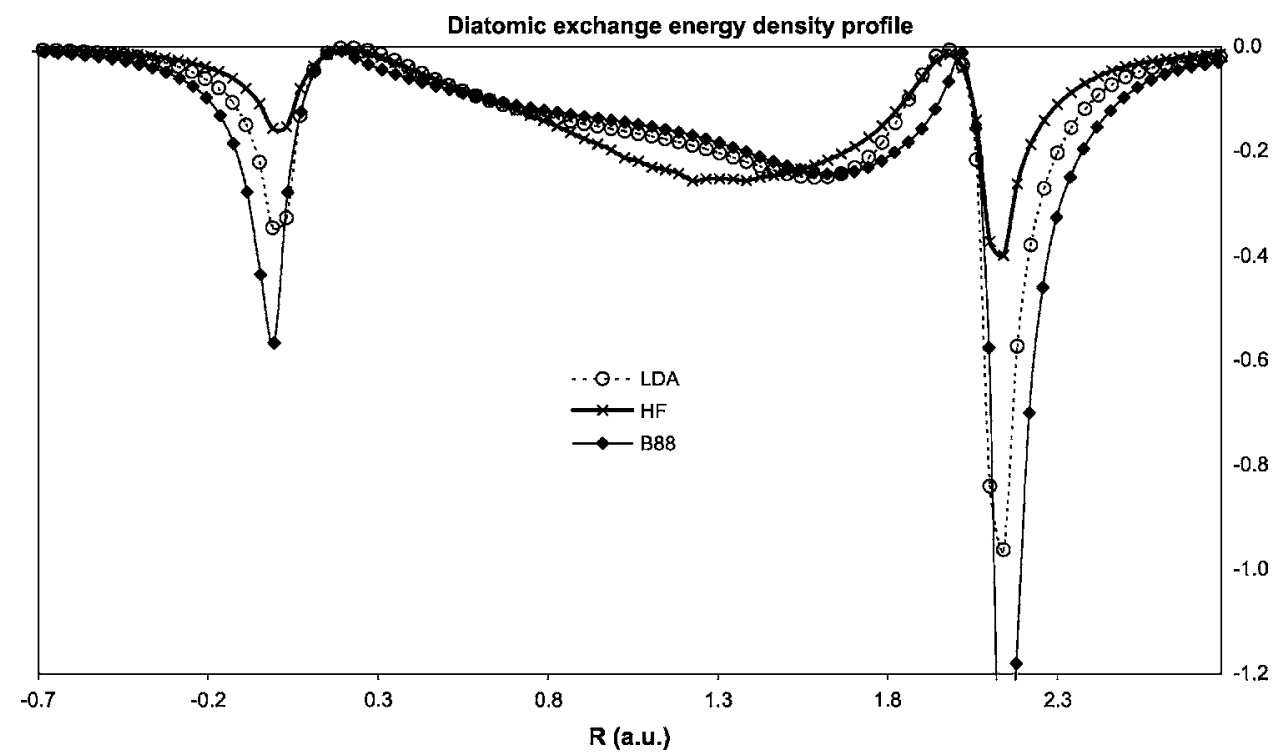

FIG. 3. Hartree-Fock and DFT diatomic exchange energy density profiles calculated from BLYP/6-31G ${ }^{* *}$ Kohn-Sham orbitals for CO molecule along the interatomic axis. The LDA and B88 values have been obtained from the bond order density (see text).

tion is almost the same at the BLYP/6-311+ $+\mathrm{G}^{* *}$ level of theory (results not reported).

We expect that a similar good correlation will be obtained by using other functionals or atomic weight function definitions such as AIM, too. Indeed, very good correlations have been obtained even by using in Eq. (22) the simplest lead-density approximation (LDA) type of exchange energy density, representing the leading term of the exchange functionals. The regression equation between the LDA and HF diatomic exchange components computed using the BLYP Kohn-Sham orbitals was $y=1.01 x+0.07$, with a correlation coefficient 0.99. Remarkably, the same equation was obtained at the HFS/6-31G ${ }^{* *}$ level of theory using the LDA Kohn-Sham orbitals and geometry. The constant underestimation of $\sim 0.07$ a.u. in the LDA results can be clearly connected with the fact that LDA exchange underestimates the energetic contribution of the atomic peaks of $\beta_{A B}(\mathbf{r})$. (Nev-

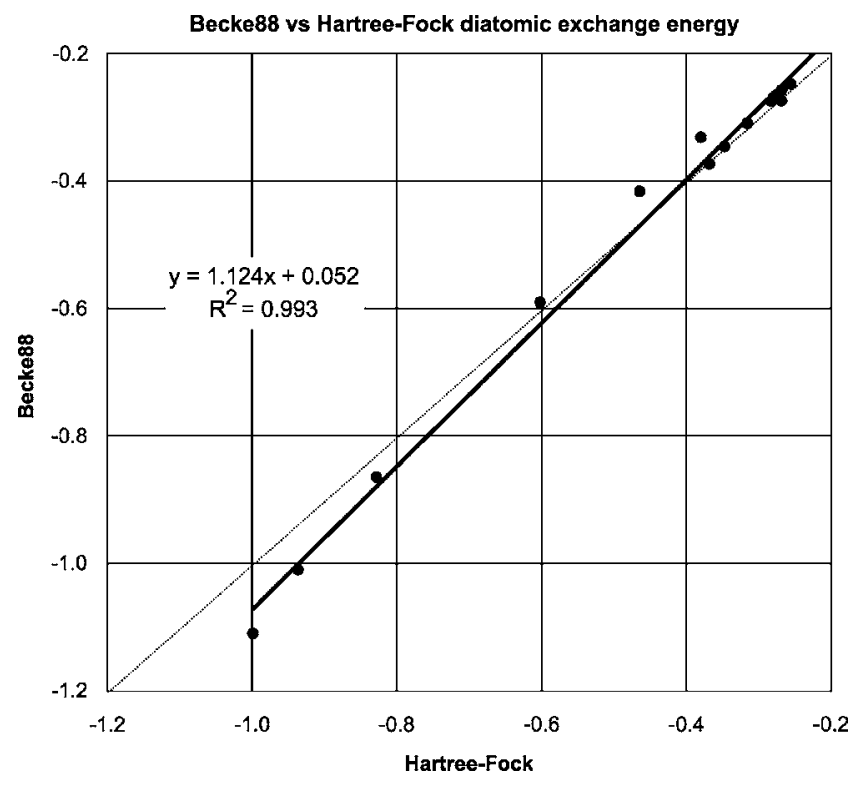

FIG. 4. Comparison of Hartree-Fock and B88 diatomic exchange energy components for the set of molecules listed in Table I using the BLYP/6-31G** Kohn-Sham orbitals. ertheless, the use of simple LDA functional may be appropriate also in the cases when the functional used cannot properly be separated into exchange and correlation terms.)

A different approach is required for calculating atomic exchange energy components. Since the exchange energy density is not a linear functional of the density, one cannot compute all the atomic and diatomic exchange energy contributions by using Eq. (22) - they would not recover the total exchange energy. (No such problem appears in the Hartree-Fock case.) Therefore, we propose to proceed in the following manner. We compute an approximate atomic exchange energy component by using Eq. (8) and then add a proper correction term to avoid double counting of the BODs. The final expression for the corrected atomic exchange component is

$$
E_{x, A}=\int w_{a}(\mathbf{r}) \varepsilon_{x}[\varrho(\mathbf{r})] d v-\frac{1}{2} \sum_{\substack{B \\(B \neq A)}} E_{x, A B} .
$$

As it is easy to see, the sum of atomic and diatomic exchange energy components $E_{x, A}$ and $E_{x, A B}$, respectively, computed in this manner, recovers the total molecular exchange energy.

Figure 5 and Table I compare the atomic exchange components calculated in the manner just discussed with the Hartree-Fock ones by using the BLYP/6-31G ${ }^{* *}$ orbitals. One can see a very good correlation for the heavy atoms and a fair one for the hydrogens. (The largest deviations are observed for the bridging hydrogens in diborane and for the hydrogen in hydrogen fluoride.)

\section{Atomic and diatomic energy components}

The reasons which lead us to calculate the diatomic exchange energy components by using the bond order density seem not applicable to the problem of correlation, so the correlation energy can be simply decomposed by using equations of type of Eq. (9), i.e., as

$$
E_{c}=\sum_{A, B} \int w_{A}(\mathbf{r}) w_{B}(\mathbf{r}) \varepsilon_{c}[\rho(\mathbf{r})] d v,
$$


Becke88 vs Hartree-Fock atomic exchange (heavy atoms)

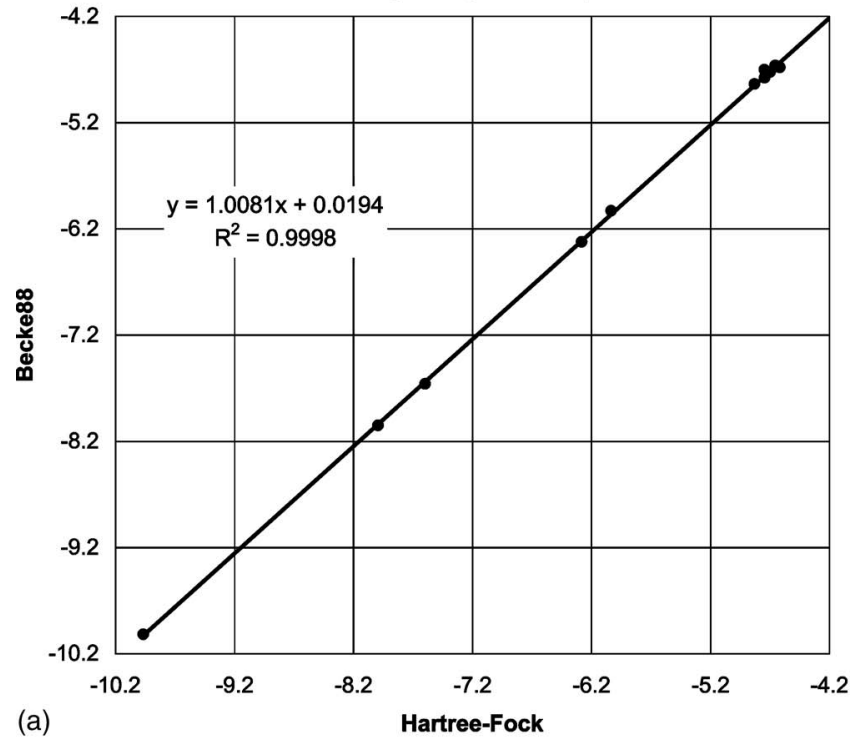

Becke88 vs Hartree-Fock atomic exchange (H atoms)

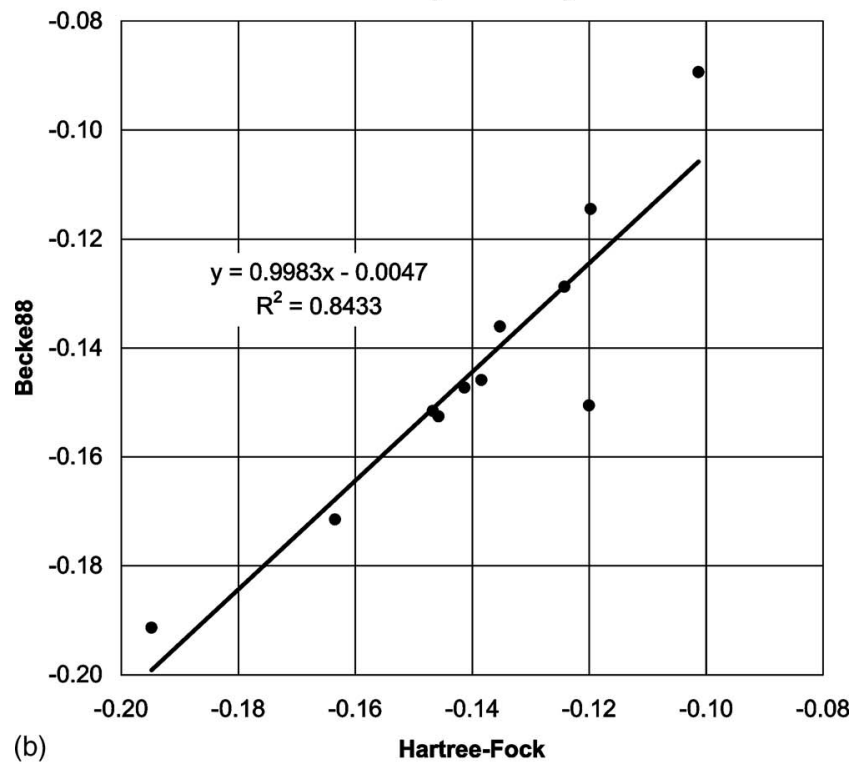

FIG. 5. (a) Hartree-Fock vs Becke 88 atomic exchange energy components for the heavy atoms of the set of molecules using the BLYP/6-31G ${ }^{* * *} \mathrm{Kohn}$ Sham orbitals. (b) Hartree-Fock vs Becke88 atomic exchange energy components for the hydrogen atoms of the set of molecules using the BLYP/6-31G** Kohn-Sham orbitals.

where $\varepsilon_{c}[\rho(\mathbf{r})]$ is the purely correlational part of the exchange-correlation energy density. This completes the formulas required to perform a complete energy decomposition in the DFT framework. For kinetic energy, we shall use Eq. (5), so we restrict ourselves to the equilibrium molecular geometries (cf. Ref. 19).

The final atomic and diatomic energy components obtained for the set of molecules at the BLYP/6-31G ${ }^{* *}$ and BLYP/6-311+ $+G^{* *}$ levels of theory are collected in Tables II and III, respectively. The diatomic BLYP/6-31G ${ }^{* *}$ values are compared to the Hartree-Fock ones using the same orbitals and geometry in Fig. 6. The correlation is again very good, especially excluding the largest values $\left(r^{2}=0.96\right)$. In

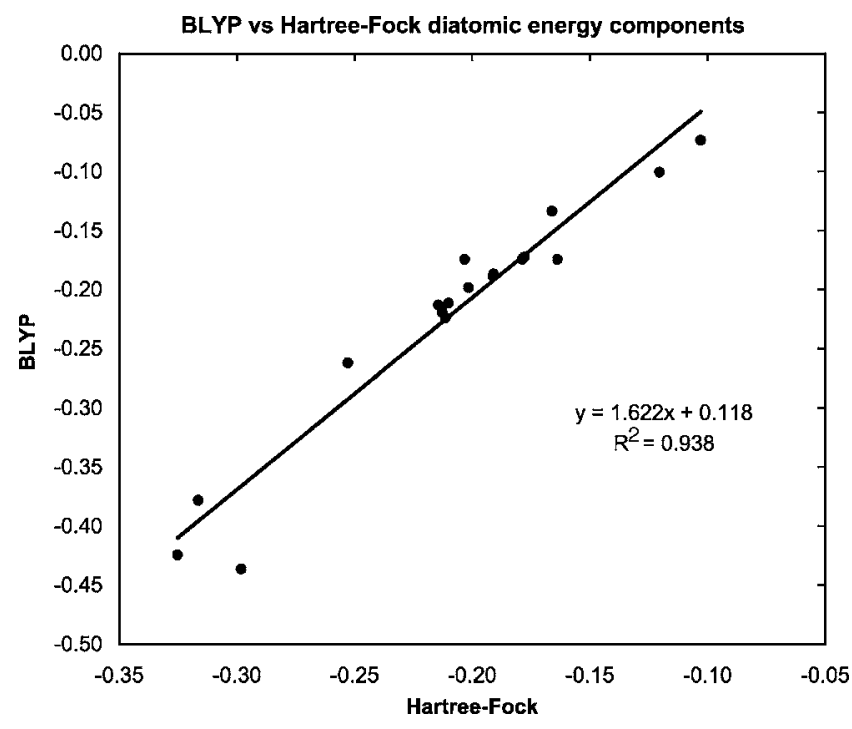

FIG. 6. Comparison of Hartree-Fock and BLYP total diatomic energy components (including correlation) for the set of molecules using the BLYP/6-31G ${ }^{* *}$ Kohn-Sham orbitals.

this case, the slope of the regression equation is greater than 1 , which is basically due to the correlation contribution. The diatomic components, like the Hartree-Fock ones in Ref. 11, are on the chemical scale and fairly independent of the basis set.

The promotion energies, defined with respect to the atomic ROBLYP energies, are, in general, within the chemical scale and very close to those obtained for the same set of molecules at the Hartree-Fock level in Ref. 11. These promotion energies are in most cases positive, even though partial ionization effects can lead to negative energy contributions on the electron-receiving atoms.

Finally, the accuracy of the numerical integration (sum of atomic and diatomic components) is slightly superior to the Hartree-Fock case. This is not surprising since it was already shown ${ }^{11}$ that the main source of error in the HartreeFock decomposition was the numerical two-electron integrations, specially the exchange ones. The main advantage of the present approach is that, relying on pure DFT exchange functionals, these cumbersome numerical integrations are avoided. That aspect may even motivate one to use the present scheme as an approximate one for analyzing the results of the Hartree-Fock calculations for rather large systems for which these two-electron integrations may become too costly.

\section{CONCLUSIONS}

In this paper, a new energy decomposition scheme is proposed for the DFT theory and is realized in the framework of the "fuzzy atoms" based 3D analysis. We had to recur to the use of bond order density when calculating diatomic exchange energy components because the diatomic contribution of exchange is related to the correlation of charge fluctuations on different atoms and thus represents an essentially nonlocal aspect even in the formally local DFT theory. By using this new concept, unexpectedly close results have been obtained for the atomic and diatomic exchange 
TABLE I. Hartree-Fock and Becke88 one and two-center exchange energy components for selected molecules at the BLYP/6-31G(d,p) level of theory.

\begin{tabular}{|c|c|c|c|c|c|c|}
\hline Molecule & Atom & $\begin{array}{c}\mathrm{HF} \\
\text { [a.u.] }\end{array}$ & $\begin{array}{c}\text { B88 } \\
\text { [a.u.] }\end{array}$ & $\begin{array}{c}\text { Atomic } \\
\text { pair }\end{array}$ & $\begin{array}{c}\mathrm{HF} \\
\text { [a.u.] }\end{array}$ & $\begin{array}{c}\text { B88 } \\
\text { [a.u.] }\end{array}$ \\
\hline $\mathrm{H}_{2}$ & $\mathrm{H}$ & -0.1948 & -0.1914 & $\mathrm{H}, \mathrm{H}$ & -0.2687 & -0.2745 \\
\hline \multirow[t]{2}{*}{$\mathrm{HF}$} & $\mathrm{H}$ & -0.1013 & -0.0894 & $\mathrm{H}, \mathrm{F}$ & -0.3682 & -0.3736 \\
\hline & $\mathrm{F}$ & -9.9662 & -10.0180 & & & \\
\hline $\mathrm{N}_{2}$ & $\mathrm{~N}$ & -6.0331 & -6.0299 & $\mathrm{~N}, \mathrm{~N}$ & -0.9986 & -1.1097 \\
\hline \multirow[t]{2}{*}{$\mathrm{CO}$} & $\mathrm{C}$ & -4.7454 & -4.7018 & $\mathrm{C}, \mathrm{O}$ & -0.9365 & -1.0097 \\
\hline & $\mathrm{O}$ & -7.5973 & -7.6595 & & & \\
\hline \multirow[t]{2}{*}{$\mathrm{H}_{2} \mathrm{O}$} & $\mathrm{O}$ & -7.9932 & -8.0508 & $\mathrm{O}, \mathrm{H}$ & -0.3469 & -0.3461 \\
\hline & $\mathrm{H}$ & -0.1197 & -0.1145 & & & \\
\hline \multirow[t]{2}{*}{$\mathrm{NH}_{3}$} & $\mathrm{~N}$ & -6.2817 & -6.3236 & $\mathrm{~N}, \mathrm{H}$ & -0.3154 & -0.3097 \\
\hline & $\mathrm{H}$ & -0.1352 & -0.1361 & & & \\
\hline \multirow[t]{2}{*}{$\mathrm{CH}_{4}$} & $\mathrm{C}$ & -4.8277 & -4.8368 & $\mathrm{C}, \mathrm{H}$ & -0.2829 & -0.2752 \\
\hline & $\mathrm{H}$ & -0.1467 & -0.1516 & & & \\
\hline \multirow[t]{2}{*}{$\mathrm{C}_{2} \mathrm{H}_{6}$} & $\mathrm{C}$ & -4.7425 & -4.7776 & $\mathrm{C}, \mathrm{C}$ & -0.3799 & -0.3315 \\
\hline & $\mathrm{H}$ & -0.1457 & -0.1526 & $\mathrm{C}, \mathrm{H}$ & -0.2738 & -0.2645 \\
\hline \multirow[t]{2}{*}{$\mathrm{C}_{6} \mathrm{H}_{6}$} & $\mathrm{C}$ & -4.6149 & -4.6812 & $\mathrm{C}, \mathrm{C}$ & -0.4646 & -0.4163 \\
\hline & $\mathrm{H}$ & -0.1384 & -0.1459 & $\mathrm{C}, \mathrm{H}$ & -0.2688 & -0.2579 \\
\hline \multirow[t]{2}{*}{$\mathrm{C}_{2} \mathrm{H}_{4}$} & $\mathrm{C}$ & -4.6973 & -4.7232 & $\mathrm{C}, \mathrm{C}$ & -0.6021 & -0.5904 \\
\hline & $\mathrm{H}$ & -0.1413 & -0.1473 & $\mathrm{C}, \mathrm{H}$ & -0.2760 & -0.2661 \\
\hline \multirow[t]{2}{*}{$\mathrm{C}_{2} \mathrm{H}_{2}$} & $\mathrm{C}$ & -4.6547 & -4.6637 & $\mathrm{C}, \mathrm{C}$ & -0.8281 & -0.8643 \\
\hline & $\mathrm{H}$ & -0.1242 & -0.1288 & $\mathrm{C}, \mathrm{H}$ & -0.2802 & -0.2698 \\
\hline \multirow[t]{3}{*}{$\mathrm{B}_{2} \mathrm{H}_{6}$} & $\mathrm{~B}$ & -3.5793 & -3.6001 & $\mathrm{~B}, \mathrm{H}_{b}$ & -0.1286 & -0.1054 \\
\hline & $\mathrm{H}_{b}$ & -0.1200 & -0.1506 & $\mathrm{~B}, \mathrm{H}_{t}$ & -0.2562 & -0.2478 \\
\hline & $\mathrm{H}_{t}$ & -0.1634 & -0.1715 & $\mathrm{~B}, \mathrm{~B}$ & -0.2236 & -0.1857 \\
\hline
\end{tabular}

TABLE II. One and two-center energy components, atomic promotion energies with respect to the atomic ROHF energies, and error of integration $\Delta$ for selected molecules at the BLYP/6-31G $(\mathrm{d}, \mathrm{p})$ level of theory.

\begin{tabular}{cccccccc}
\hline \hline \multirow{2}{*}{ Molecule } & Atom & $\begin{array}{c}\Delta \mathrm{E}_{A} \mathrm{HF} \\
{[\mathrm{kcal} / \mathrm{mol}]}\end{array}$ & $\begin{array}{c}\Delta \mathrm{E}_{A} \mathrm{BLYP} \\
{[\mathrm{kcal} / \mathrm{mol}]}\end{array}$ & $\begin{array}{c}\text { Atomic } \\
\text { pair }\end{array}$ & $\begin{array}{c}\mathrm{E}_{A B} \mathrm{HF} \\
{[\mathrm{kcal} / \mathrm{mol}]}\end{array}$ & $\begin{array}{c}\mathrm{E}_{A B} \mathrm{BLYP} \\
{[\mathrm{kcal} / \mathrm{mol}]}\end{array}$ & $\begin{array}{c}\Delta \\
{[\mathrm{kcal} / \mathrm{mol}]}\end{array}$ \\
\hline $\mathrm{H}_{2}$ & $\mathrm{H}$ & 9.2 & -0.8 & $\mathrm{H}, \mathrm{H}$ & -102.8 & -109.5 & -0.2 \\
$\mathrm{HF}$ & $\mathrm{H}$ & 28.6 & 27.6 & $\mathrm{H}, \mathrm{F}$ & -132.7 & -140.6 & -2.1 \\
& $\mathrm{~F}$ & 10.3 & -23.9 & & & & \\
$\mathrm{~N}_{2}$ & $\mathrm{~N}$ & 42.5 & 18.5 & $\mathrm{~N}, \mathrm{~N}$ & -187.1 & -273.9 & -0.7 \\
$\mathrm{CO}$ & $\mathrm{C}$ & -43.2 & -32.4 & $\mathrm{C}, \mathrm{O}$ & -204.1 & -266.4 & 1.6 \\
& $\mathrm{O}$ & 82.1 & 38.2 & & & & \\
$\mathrm{H}_{2} \mathrm{O}$ & $\mathrm{O}$ & 63.0 & 7.0 & $\mathrm{O}, \mathrm{H}$ & -133.5 & -137.6 & 0.6 \\
& $\mathrm{H}$ & 23.5 & 17.1 & & & & \\
$\mathrm{NH}_{3}$ & $\mathrm{~N}$ & 116.1 & 47.1 & $\mathrm{~N}, \mathrm{H}$ & -131.8 & -132.7 & -0.2 \\
& $\mathrm{H}$ & 23.7 & 12.5 & & & & \\
$\mathrm{CH}_{4}$ & $\mathrm{C}$ & 67.2 & 27.7 & $\mathrm{C}, \mathrm{H}$ & -134.6 & -133.7 & 0.3 \\
& $\mathrm{H}$ & 27.7 & 13.6 & & & & \\
$\mathrm{C}_{2} \mathrm{H}_{6}$ & $\mathrm{C}$ & 70.3 & 17.8 & $\mathrm{C}, \mathrm{C}$ & -104.2 & -83.8 & -1.9 \\
& $\mathrm{H}$ & 33.1 & 17.6 & $\mathrm{C}, \mathrm{H}$ & -126.5 & -124.4 & \\
$\mathrm{C}_{6} \mathrm{H}_{6}$ & $\mathrm{C}$ & 57.8 & -7.5 & $\mathrm{C}, \mathrm{C}$ & -127.5 & -109.4 & -1.3 \\
& $\mathrm{H}$ & 37.9 & 22.3 & $\mathrm{C}, \mathrm{H}$ & -111.6 & -108.2 & \\
$\mathrm{C}_{2} \mathrm{H}_{4}$ & $\mathrm{C}$ & 50.9 & 8.4 & $\mathrm{C}, \mathrm{C}$ & -158.7 & -164.5 & -2.6 \\
& $\mathrm{H}$ & 32.8 & 18.2 & $\mathrm{C}, \mathrm{H}$ & -119.8 & -117.3 & \\
$\mathrm{C}_{2} \mathrm{H}_{2}$ & $\mathrm{C}$ & 38.2 & 10.4 & $\mathrm{C}, \mathrm{C}$ & -198.5 & -237.3 & -1.6 \\
& $\mathrm{H}$ & 34.5 & 21.9 & $\mathrm{C}, \mathrm{H}$ & -112.2 & -109.3 & \\
$\mathrm{~B}_{2} \mathrm{H}_{6}$ & $\mathrm{~B}$ & 26.7 & -7.6 & $\mathrm{~B}, \mathrm{H}_{b}$ & -75.6 & -63.1 & -3.7 \\
& $\mathrm{H}$ & 66.8 & 36.8 & $\mathrm{~B}, \mathrm{H}{ }_{t}$ & -119.9 & -118.2 & \\
& $\mathrm{H}$ & 32.9 & 15.9 & $\mathrm{~B}, \mathrm{~B}$ & -64.6 & -46.0 & \\
\hline \hline & & & & & & &
\end{tabular}


TABLE III. One and two-center energy components, atomic promotion energies with respect to the atomic ROHF energies, and error of integration $\Delta$ for selected molecules at the BLYP/6 $311++G(d, p)$ level of theory.

\begin{tabular}{|c|c|c|c|c|c|c|c|}
\hline Molecule & Atom & $\begin{array}{c}\Delta \mathrm{E}_{A} \mathrm{HF} \\
{[\mathrm{kcal} / \mathrm{mol}]}\end{array}$ & $\begin{array}{l}\Delta \mathrm{E}_{A} \text { BLYP } \\
{[\mathrm{kcal} / \mathrm{mol}]}\end{array}$ & $\begin{array}{l}\text { Atomic } \\
\text { pair }\end{array}$ & $\begin{array}{c}\mathrm{E}_{A B} \mathrm{BLYP} \\
\text { [a.u.] }\end{array}$ & $\begin{array}{l}\mathrm{E}_{A B} \text { BLYP } \\
{[\mathrm{kcal} / \mathrm{mol}]}\end{array}$ & $\begin{array}{c}\Delta \\
{[\mathrm{kcal} / \mathrm{mol}]}\end{array}$ \\
\hline $\mathrm{H}_{2}$ & $\mathrm{H}$ & 10.1 & 0.3 & $\mathrm{H}, \mathrm{H}$ & -102.7 & -109.8 & -0.2 \\
\hline \multirow[t]{2}{*}{$\mathrm{HF}$} & $\mathrm{H}$ & 32.8 & 33.3 & $\mathrm{H}, \mathrm{F}$ & -134.3 & -143.8 & -1.3 \\
\hline & $\mathrm{F}$ & 11.7 & -31.2 & & & 0.0 & \\
\hline $\mathrm{N}_{2}$ & $\mathrm{~N}$ & 47.5 & 23.0 & $\mathrm{~N}, \mathrm{~N}$ & -193.0 & -283.6 & 0.1 \\
\hline \multirow[t]{2}{*}{$\mathrm{CO}$} & $\mathrm{C}$ & -46.3 & -36.7 & $\mathrm{C}, \mathrm{O}$ & -207.8 & -273.5 & 1.2 \\
\hline & $\mathrm{O}$ & 93.8 & 50.2 & & & & \\
\hline \multirow[t]{2}{*}{$\mathrm{H}_{2} \mathrm{O}$} & $\mathrm{O}$ & 62.0 & -0.9 & $\mathrm{O}, \mathrm{H}$ & -136.3 & -141.2 & 0.6 \\
\hline & $\mathrm{H}$ & 28.4 & 22.7 & & & & \\
\hline \multirow[t]{2}{*}{$\mathrm{NH}_{3}$} & $\mathrm{~N}$ & 112.8 & 37.5 & $\mathrm{~N}, \mathrm{H}$ & -133.9 & -134.5 & 0.2 \\
\hline & $\mathrm{H}$ & 27.7 & 17.0 & & & & \\
\hline \multirow[t]{2}{*}{$\mathrm{CH}_{4}$} & $\mathrm{C}$ & 59.0 & 18.0 & $\mathrm{C}, \mathrm{H}$ & -134.4 & -133.7 & 1.0 \\
\hline & $\mathrm{H}$ & 32.3 & 18.0 & & & & \\
\hline \multirow[t]{2}{*}{$\mathrm{C}_{2} \mathrm{H}_{6}$} & $\mathrm{C}$ & 69.1 & 15.3 & $\mathrm{C}, \mathrm{C}$ & -109.2 & -89.9 & -0.9 \\
\hline & $\mathrm{H}$ & 37.2 & 21.6 & $\mathrm{C}, \mathrm{H}$ & -126.5 & -124.7 & \\
\hline \multirow[t]{2}{*}{$\mathrm{C}_{6} \mathrm{H}_{6}$} & $\mathrm{C}$ & 64.4 & -1.2 & $\mathrm{C}, \mathrm{C}$ & -132.7 & -114.5 & 0.0 \\
\hline & $\mathrm{H}$ & 41.0 & 25.6 & $\mathrm{C}, \mathrm{H}$ & -112.0 & -108.7 & \\
\hline \multirow[t]{2}{*}{$\mathrm{C}_{2} \mathrm{H}_{4}$} & $\mathrm{C}$ & 51.3 & 7.1 & $\mathrm{C}, \mathrm{C}$ & -164.5 & -170.6 & -2.0 \\
\hline & $\mathrm{H}$ & 36.6 & 22.2 & $\mathrm{C}, \mathrm{H}$ & -119.8 & -117.6 & \\
\hline \multirow[t]{2}{*}{$\mathrm{C}_{2} \mathrm{H}_{2}$} & $\mathrm{C}$ & 38.4 & 9.4 & $\mathrm{C}, \mathrm{C}$ & -203.0 & -242.8 & 1.9 \\
\hline & $\mathrm{H}$ & 38.2 & 25.9 & $\mathrm{C}, \mathrm{H}$ & -111.1 & -106.6 & \\
\hline \multirow[t]{3}{*}{$\mathrm{B}_{2} \mathrm{H}_{6}$} & B & 23.8 & -12.6 & $\mathrm{~B}, \mathrm{H}_{b}$ & -73.4 & -60.9 & -3.7 \\
\hline & $\mathrm{H}_{b}$ & 68.0 & 38.3 & $\mathrm{~B}, \mathrm{H}_{t}$ & -119.1 & -117.8 & \\
\hline & $\mathrm{H}_{t}$ & 35.7 & 18.8 & $\mathrm{~B}, \mathrm{~B}$ & -66.9 & -48.5 & \\
\hline
\end{tabular}

energy components to the "exact" (Hartree-Fock) ones calculated by using the same Kohn-Sham orbitals. As a consequence, very reasonable overall energy components have been obtained, too.

\section{ACKNOWLEDGMENTS}

This work has been partly supported by the SpanishHungarian Joint Project No. HH2004-0010 (TÉT E18/2004), as well as by the Hungarian Research Fund (Grant No. OTKA T43558) and the Spanish MEC Project No. CTQ2005-02698/BQU.

${ }^{1}$ There are hybrid schemes (Refs. 2 and 3), too, in which only the overlap integral matrix of the basis orbitals is decomposed into atomic contributions by using a 3D analysis, and then this decomposition is utilized in that or another manner to decompose the energy formula. We think that a truly $3 \mathrm{D}$ analysis should not explicitly make use of the basis orbitalswhich does not mean that the hybrid schemes may not be useful.

${ }^{2}$ I. Mayer and A. Hamza, Int. J. Quantum Chem. 103, 798 (2005).

${ }^{3}$ D. R. Alcoba, A. Torre, L. Lain, and R. C. Bochicchio, J. Chem. Phys. 12, 074102 (2005).

${ }^{4}$ I. Mayer, Chem. Phys. Lett. 332, 381 (2000).

${ }^{5}$ I. Mayer, Chem. Phys. Lett. 382, 265 (2003).

${ }^{6}$ S. F. Vyboishchikov, P. Salvador, and M. Duran, J. Chem. Phys. 122, 244110 (2005)

${ }^{7}$ R. F. W. Bader, Atoms in Molecules: A Quantum Theory (Oxford University Press, Oxford, 1990).

${ }^{8}$ P. Salvador, M. Duran, and I. Mayer, J. Chem. Phys. 115, 1153 (2001).

${ }^{9}$ M. A. Blanco, A. Martín Pendás, and E. Francisco, J. Chem. Theory Comput. 1, 1096 (2005).

${ }^{10}$ I. Mayer and A. Hamza, Theor. Chem. Acc. 105, 360 (2001).

${ }^{11}$ P. Salvador and I. Mayer, J. Chem. Phys. 120, 5046 (2004).

${ }^{12}$ I. Mayer and P. Salvador, Chem. Phys. Lett. 383, 368 (2004).

${ }^{13}$ F. L. Hirshfeld, Theor. Chim. Acta 44, 129 (1977).

${ }^{14}$ A. E. Clark and E. R. Davidson, J. Chem. Phys. 115, 7382 (2001).

${ }^{15}$ A. E. Clark and E. R. Davidson, Int. J. Quantum Chem. 93, 384 (2003).
${ }^{16}$ A. D. Becke, J. Chem. Phys. 88, 2547 (1988).

${ }^{17}$ In the "fuzzy atom" scheme, the kinetic energy can be presented as a sum of either atomic contribution or that of atomic and diatomic components, while in the AIM case, the disjunct character of the atomic domains permits only its decomposition into atomic contributions. They are strongly positive; thus the AIM results gave pretty big atomic "promotions" and, accordingly, somewhat too negative bonding energy components-as compared to the bond dissociation energies.

${ }^{18}$ I. Mayer, Phys. Chem. Chem. Phys. 8, 4630 (2006).

${ }^{19}$ I. Mayer, Faraday Discuss. 135, 439 (2007).

${ }^{20}$ Operators $\hat{\rho}_{A}$, in general, need not be Hermitian; those considered in this paper, however, are.

${ }^{21}$ I. Mayer, Simple Theorems, Proofs, and Derivations in Quantum Chemistry (Kluwer Academic/Plenum Publishers, New York etc., 2003).

${ }^{22}$ These relationships are due to the fact that for single determinant wave functions, the second order density matrix can be expressed via the first order one: $\rho_{2}\left(x_{1}, x_{2}, x_{1}^{\prime}, x_{2}^{\prime}\right)=\rho_{1}\left(x_{1}, x_{1}^{\prime}\right) \rho_{1}\left(x_{2}, x_{2}^{\prime}\right)-\rho_{1}\left(x_{1}, x_{2}^{\prime}\right) \rho_{1}\left(x_{2}, x_{1}^{\prime}\right)$.

${ }^{23}$ For the discussion of an analogous relationship between Hilbert-space bond orders, diatomic exchange densities, and exchange energy components, see Ref. 24

${ }^{24}$ I. Mayer, J. Phys. Chem. B 111, 669 (2007).

${ }^{25}$ N. P. Borisova and S. G. Semenov, Vestn. Leningr. Univ., Ser. 4: Fiz., Khim. 16, 119 (1973).

${ }^{26}$ K. A. Wiberg, Tetrahedron 24, 1083 (1968).

${ }^{27}$ I. Mayer, J. Comput. Chem. 28, 204 (2007)

${ }^{28}$ Actually, it has been shown analytically that for the homonuclear diatomics of the first row elements (except $\mathrm{C}_{2}$ having a peculiar electronic structure) treated at the CNDO or minimal basis ab initio level, the bond order index equals the "chemist's bond order" $B=\frac{1}{2}\left(N_{\text {bond }}-N_{\text {antibond }}\right)$, where $N_{\text {bond }}$ and $N_{\text {antibond }}$ are the numbers of electrons occupying bonding and antibonding orbitals, respectively (Refs. 25 and 27).

${ }^{29}$ M. S. de Giambiagi, M. Giambiagi, and F. E. Jorge, Theor. Chim. Acta 68, 337 (1985).

${ }^{30}$ H. Fischer and H. Kollmar, Theor. Chim. Acta 16, 163 (1970).

${ }^{31}$ I. Mayer, Chem. Phys. Lett. 97, 270 (1983).

${ }^{32}$ I. Mayer, Int. J. Quantum Chem. 23, 341 (1983).

${ }^{33}$ The name "self-charge" has also been proposed for the integral of this quantity (Ref. 29); however, we prefer not to use that term because another definition in terms of second quantized operators was also simulta- 
neously used for the "self charge," and the two definitions were different due to an error; this led to a polemic in the literature (Refs. 34-36).

${ }^{34}$ F. E. Jorge and A. B. Batista, Chem. Phys. Lett. 138, 115 (1987).

${ }^{35}$ M. S. de Giambiagi and M. Giambiagi, Chem. Phys. Lett. 141, 466 (1987).

${ }^{36}$ I. Mayer, Chem. Phys. Lett. 148, 95 (1988).

${ }^{37}$ E. Matito, M. Sola, P. Salvador, and M. Duran, Faraday Discuss. 135, 325 (2007).

${ }^{38}$ E. Francisco, A. Martin-Pendas, and M. A. Blanco, J. Chem. Theory Comput. 2, 90 (2006).
${ }^{39}$ The one-dimensional cross sections on Fig. 3 make the wrong impression that the DFT exchange energies are much larger in absolute value than the Hartree-Fock one. This is not the case; however, their integrals are very close-as it will be discussed below in detail.

${ }^{40}$ Density Functional Repository as developed and distributed by the Quantum Chemistry Group, CCLRC Daresbury Laboratory, Daresbury, Cheshire, WA4 4AD United Kingdom. Contact Huub van Dam at h.j.j.vandam@dl.ac.uk or Paul Sherwood for further information.

${ }^{41}$ In Refs. 11 and 12, it was called Chebyshev integration by mistake. 